\title{
Should the "elephant trunk" be skeletonized? Total arch replacement combined with stented elephant trunk implantation for Stanford type A aortic dissection
}

\author{
Zhi-Gang Liu, MD, Li-Zhong Sun, MD, Qian Chang, MD, Jun-Ming Zhu, MD, Chao Dong, MD, Chun-Tao Yu, MD, \\ Yong-Min Liu, MD, and Hai-Tao Zhang, MD
}

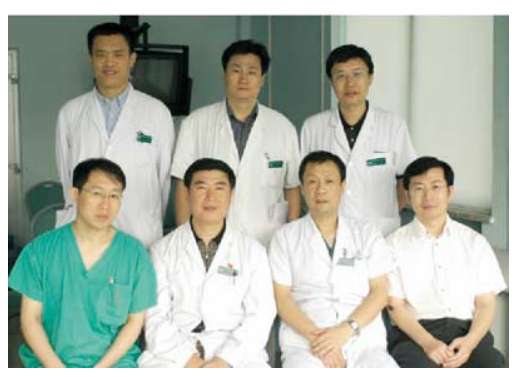

From the Department of Cardiovascular Surgery, Cardiovascular Institute and $\mathrm{Fu}$ wai Hospital, Chinese Academe of Medical Science and Peking Union Medical College, Beijing, China.

Read at the Eighty-fifth Annual Meeting of The American Association for Thoracic Surgery, San Francisco, Calif, April 10-13, 2005.

Received for publication April 29, 2005; revisions received Aug 24, 2005; accepted for publication Sept 9, 2005.

Address for reprints: Li-Zhong Sun, MD, Department of Cardiovascular Surgery, Cardiovascular Institute and Fuwai Hospital Beijing, CAMS \& PUMC, 100037, 167 Bei Li Shi Rd, Xi Cheng District, Beijing, Peoples Republic of China (E-mail: liuzgfwh@ hotmail.com).

J Thorac Cardiovasc Surg 2006;131:107-13 $0022-5223 / \$ 32.00$

Copyright () 2006 by The American Association for Thoracic Surgery

doi:10.1016/j.jtcvs.2005.09.015
Objectives: To eliminate the residual false lumen in the descending thoracic aorta and improve long-term outcomes of surgical intervention for Stanford type A aortic dissection, we performed the skeletonized "elephant trunk" procedure in the ascending aorta and aortic arch replacement combined with transaortic stented graft implantation into the descending aorta for both acute and chronic type A aortic dissection, and the short-term results were compared.

Methods: Between April 2003 and November 2004, 60 consecutive patients (mean age, $53 \pm 16.7$; approximate range, 28-78 years) with acute $(\mathrm{n}=36)$ or chronic $(\mathrm{n}=$ 24) type A aortic dissection underwent this procedure. Right axillary artery cannulation was used for cardiopulmonary bypass and selected cerebral perfusion. The stented graft, a 10-cm-long woven Dacron graft with a self-expandable stent, was implanted through the aortic arch during hypothermic circulatory arrest. Enhanced electric beam computed tomography was performed in each patient before discharge, 3 months after the operation, and once each year thereafter to evaluate the postoperative time course of the residual false lumen.

Results: Cardiopulmonary bypass time was $166 \pm 38$ minutes, and average selective cerebral perfusion and lower body arrest time was $30 \pm 15$ minutes. The in-hospital mortality was $3.3 \%(2 / 60)$. Thrombus obliteration of the residual false lumen in the descending thoracic aorta was observed in $92 \%$ and $85 \%$ of the acute and chronic aortic dissections, respectively, 3 months postoperatively. There was no late death during follow-up.

Conclusions: The skeletonized elephant trunk procedure is an effective way of closing the residual false lumen of the descending aorta and might contribute to better long-term outcomes for both acute and chronic type A aortic dissection.

I n China aortic dissection is a very common category of the aortic diseases. According to unpublished data from the epidemic institute of the Chinese Academy of Medical Science, the incidence of aortic dissection could be as high as 20 to 50 per million townsmen. The conventional treatment for acute type A aortic dissection used to be replacement of the ascending portion of the aorta. Because the dissection involves the entire aorta, the pathology of residual false lumen of the downstream aorta plays an essential role in determination of prognosis. As long as the false lumen remains patent, the chances of survival might remain in hazard, and reoperation is inevitable. Therefore a method that can extensively replace the dissected aorta with minimal morbidity and that can eliminate the need for additional operations is required. Kato and coworkers ${ }^{1}$ developed a hybrid technique in which the ascending aorta and aortic arch were replaced with a branched graft, and a stented graft was implanted into the descending aorta for the treatment of acute type A aortic dissection. Enlightened by this technique, we 


\section{Abbreviations and Acronyms \\ $\mathrm{CPB}=$ cardiopulmonary bypass \\ $\mathrm{EBCT}=$ electric beam computed tomography \\ $\mathrm{SCP}=$ selective cerebral perfusion}

proposed the skeletonized "elephant trunk" procedure for the treatment of both acute and chronic type A aortic dissection and had good results. Herein we report the outcomes of our experience and the problems we encountered.

\section{Patients and Methods \\ Patient Profile}

Between April 2003 and November 2004, 60 consecutive patients with acute $(\mathrm{n}=36)$ or chronic $(\mathrm{n}=24)$ Stanford type A aortic dissection underwent ascending aorta and total aortic arch replacement combined with transaortic stented graft implantation into the descending aorta: the skeletonized elephant trunk procedure. The diagnosis was made on the basis of electric beam computed tomography (EBCT), magnetic resonance imaging, and echocardiography. Fifty-four patients were male, and 6 were female, with a mean age of $53 \pm 16.7$ years (approximate range, 28-78 years). Acute dissection was diagnosed in 36 patients, whereas chronic dissection was observed in 24 patients. Nine patients showed classic Marfan syndrome, and 7 had a history of coronary artery disease. Forty-six patients had hypertension without receiving effective antihypertensive treatment. A history of stroke was noted in 2 patients. Other preoperative complications related to the aortic dissection included aortic regurgitation in 39 patients, cardiac tamponade in 15 patients, acute renal dysfunction in 2 patients, transient cerebral ischemia in 9 patients, and coronary ischemia in 7 patients (Table 1). Five patients had a history of initial aortic operations, 2 had composite valved graft implantation, 2 had intraluminal stent graft implantation, and 1 had ascending aorta replacement.

The interval between the onset of pain and surgical intervention was $6.8 \pm 4.5$ days in acute cases and $42.5 \pm 20$ days (those with a history $<1$ year) and $3.3 \pm 4.3$ years (there were 7 patients with a history $>1$ year) in chronic cases.

In this series the location of the primary intimal tears were as follows. In the acute group there were 11 in the ascending aorta, 16 in the descending aorta, and 4 in the arch, and multiple tears were present in 5 patients. In the chronic group there were 8 in the ascending aorta, 7 in the descending aorta, and 5 in the arch, and there were multiple tears in 4 patients.

\section{Stented Graft and Branched Graft}

The stented graft is $10 \mathrm{~cm}$ long and 28 to $32 \mathrm{~mm}$ in diameter. It consists of a Gianturco-type self-expandable metallic stent (Microport Medical Corp) and a high-porosity woven Dacron graft (Intervascular OLP, Intervascular Inc). At each end, there is a 10 -mm-long stent-free sewing edge. The synthetic branched vascular graft ( 28 to $32 \mathrm{~mm}$ in diameter) was a product of InterGuard, Intervascular, Datascope Company.

It is very important to determine the right size of a stent graft. For those acute cases, the diameter of a selected stent graft should
TABLE 1. Patient data

\begin{tabular}{lc}
\hline & No. of patients \\
\hline All patients & 60 \\
Female & 6 \\
Male & 54 \\
Acute dissection & 36 \\
Chronic dissection & 24 \\
Aortic insufficiency & 39 \\
Cardiac tamponade & 15 \\
Marfan syndrome & 9 \\
Coronary disease & 7 \\
Cerebrovascular disease & 2 \\
Hypertension & 46 \\
Renal dysfunction & 2 \\
Diabetes & 2 \\
\hline
\end{tabular}

be $10 \%$ bigger than the diameter of the native proximal descending aorta. For chronic cases, we determine the size of an implanted stent graft according to the size of the true lumen; the diameter of the native proximal descending aorta also needs to be taken into consideration. The size of a chosen stent graft should be $10 \%$ to $20 \%$ bigger than the size of the true lumen. The most preferable size of stent graft we used was approximately 28 to $30 \mathrm{~mm}$ for both acute and chronic cases.

\section{Operative Technique}

After induction of general anesthesia, the arterial blood pressures of both the upper and lower limbs were monitored. A right subclavian incision was made, and the right axillary artery was exposed. Then a median sternotomy was performed. An arterial cannula was inserted into the right axillary artery, and a dual-stage atriocaval cannula was placed at the right atrium. Right axillary artery cannulation was routinely used for cardiopulmonary bypass (CPB) and selected cerebral perfusion.

The arterial line was bifurcated, one for axillary artery perfusion and the other for perfusing branch cannulation and perfusion, as shown in Figure 1. The CPB flow was maintained between 2.2 and $2.4 \mathrm{~L} \cdot \min ^{-1} \cdot \mathrm{m}^{-2}$, and patients were cooled to a rectal temperature of approximately $18^{\circ} \mathrm{C}$ to $20^{\circ} \mathrm{C}$. Cold blood cardioplegia was routinely administered for myocardial protection. During cooling, the brachiocephalic arteries were dissociated and exposed as long as possible. The ascending aorta was clamped at the distal end, the aorta just distal to the sinotubular junction was transected, and proximal manipulations, such as aortic valve repair, sinus of Valsalva reconstruction, coronary artery bypass grafting, and composite valved graft replacement, were commenced if necessary. CPB was discontinued when the rectal temperature was less than $20^{\circ} \mathrm{C}$, while the brain continued to be perfused at a rate of approximately 5 to $10 \mathrm{~mL} \cdot \mathrm{kg}^{-1} \cdot \mathrm{min}^{-1}$ through the right axillary artery cannula. The transverse aortic arch was transected between the left common carotid and left subclavian arteries. A catheter sheath containing the stented graft was inserted into the appropriate segment of the descending aorta, and the stented graft was deployed by using the pulling rod. The intima is very fragile in acute cases, and great care must be taken to prevent creating a new intimal tear when 


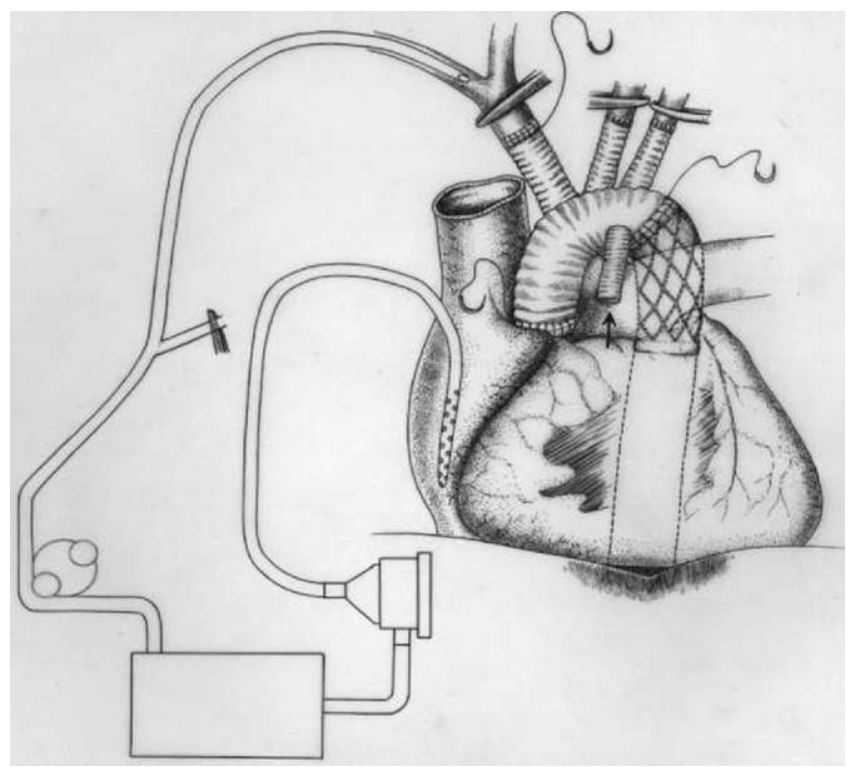

Figure 1. Diagram of the skeletonized elephant trunk technique.

performing stent graft implantation. After the graft was positioned, the stent graft was expanded with a balloon expander if necessary. The distal end of the branched graft was anastomosed to the descending aorta or transected transverse aortic arch containing the intraluminal stented graft. As soon as the anastomosis was finished, antegrade systemic perfusion was begun through the perfusion branch connected to the arterial line of the CPB circuit, and then the brachiocephalic arteries were anastomosed to the side branches of the branched graft. The brachiocephalic arteries were then reperfused through both right axillary cannulation and the perfusion branch of the branched graft, which was connected to the CPB circuit. When the left common carotid artery anastomosis was finished and reperfused, rewarming was started. Finally, the proximal end of the branched graft was anastomosed to the ascending aorta. The diagram of the skeletonized elephant trunk technique is shown in Figure 1.

Only 2 anastomoses need to be made during the selective cerebral perfusion (SCP) period: first a distal anastomosis (to the descending aorta) and then a left common carotid artery anastomosis to the branched graft. After these 2 anastomoses were accomplished, SCP was discontinued, CPB was resumed to normal flow gradually, and rewarming was started. Then we performed left subclavian artery and innominate artery anastomosis, and finally, proximal anastomosis (to the proximal ascending aorta) was performed. Thus at least 20 to 30 minutes of SCP time can be saved.

There should not be any gradient of arterial blood pressure between the upper and lower limbs after the stented elephant trunk procedure is finished, and this is crucial. If the arterial blood pressure gradient between the lower and upper limbs was more than $30 \mathrm{~mm} \mathrm{Hg}$ accompanied by a dramatic urine output decrease, fenestration of the septum might be indicated.
TABLE 2. Concomitant procedures

\begin{tabular}{lc}
\hline & No. of patients \\
\hline Standard & 36 \\
Bentall technique & 9 \\
CABG & 4 \\
AV repair & 4 \\
AV repair + CABG & 3 \\
Modified David procedure & 1 \\
Sinus of Valsalva reconstruction & 3
\end{tabular}

Standard, Ascending aorta and total arch replacement plus stented graft implantation; $C A B G$, coronary artery bypass grafting; $A V$, aortic valve; modified David procedure, aortic root replacement with aortic valvesparing technique.

In 12 patients we transected and closed the left subclavian artery at its ostium to make the distal anastomosis line much closer. If the left subclavian artery was too deep inside the operative field, we occluded its ostium with the stented graft and ligated it at its proximal end and anastomosed the graft branch to the left axillary artery in an end-to-side fashion.

\section{Follow-up}

EBCT was performed in each patient before discharge, 3 months after surgical intervention, and once each year after discharge to evaluate the postoperative time course of the residual false lumen (aortoangiography was administered if necessary). All patients discharged from the hospital were followed up to the end date of the study (December 2004). The patients were contacted by telephone or direct interview in our outpatient clinic.

\section{Results \\ Operative Data}

The mean CPB time was $166 \pm 38$ minutes, aortic crossclamp time was $110 \pm 26$ minutes, average SCP time was $30 \pm 15$ minutes, and lower body arrest time was $20 \pm 6$ minutes. Nasopharyngeal temperature decreased to approximately $15^{\circ} \mathrm{C}$ to $20^{\circ} \mathrm{C}$ for the period of lower body arrest and SCP.

Concomitant procedures are shown in Table 2: the Bentall procedure in 9 patients, aortic valve repair in 7 patients (not including commissural resuspension), aortic valve replacement in 2 patients, sinus of Valsalva reconstruction in 3 patients, coronary artery bypass grafting in 7 patients, and the modified David procedure in 1 patient. Five patients had a history of initial aortic surgery: composite valved graft implantation in 2 patients, ascending aorta replacement in 1 patient, and endovascular stent graft implantation and failure in 2 patients.

\section{Mortality}

There were 2 in-hospital deaths in this group. One patient who exhibited acute renal failure preoperatively died of multiorgan failure 2 weeks after the operation, and the other, who had brain infarction 2 years preoperatively, died 

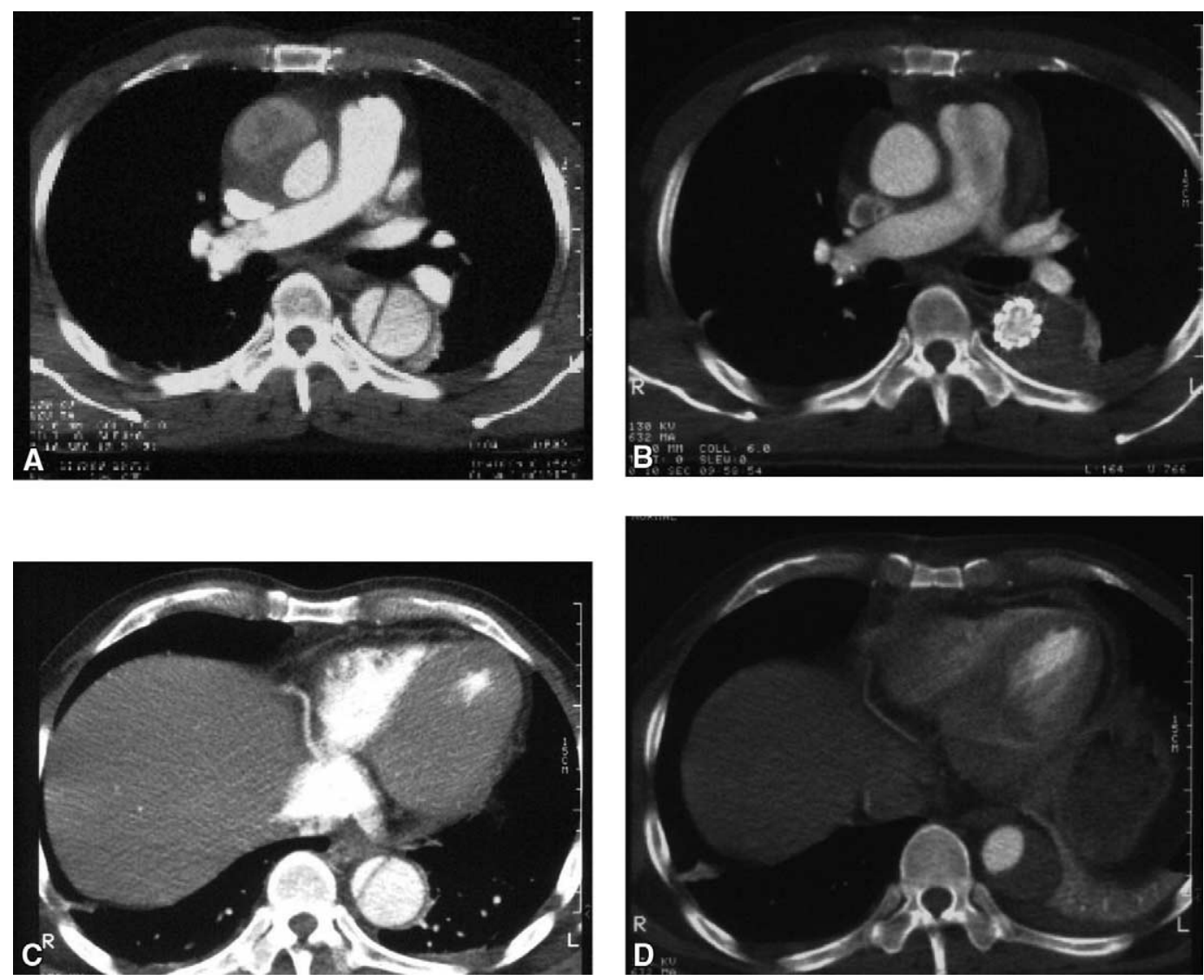

Figure 2. Electric beam computed tomography of a chronic case of type A aortic dissection before the operation, in which the primary intimal tear was located in the arch. The false channel in the ascending aorta was filled with thrombus (A), and the true lumen was compressed severely in the diaphragmatic level (C). B and D, Electric beam computed tomography of the same patient 2 weeks after surgical intervention shows that the true channel in the descending aorta was resumed, and the false channel was closed with thrombus.

of extensive cerebral infarction (brain death) 2 months after the operation.

\section{Morbidity}

Three patients underwent reoperations because of bleeding from the anastomotic site of the distal aortic stump in 2 patients and the proximal aortic stump in 1 patient. Cerebral infarction was observed in 3 patients, among whom one recovered completely without any neurologic deficit, one exhibited monoplegia of his right arm, and the other died as mentioned above. Paraplegia occurred in 1 patient. Temporary hemodialysis was required in 3 patients who exhibited acute renal failure preoperatively. Two of them fully recovered, and 1 patient died of multiorgan failure (as mentioned previously). Transient neurologic dysfunction was observed in 8 patients, who were fully recovered within 1 week. Prolonged ( $>3$ days) mechanical ventilation support was needed in 5 patients.

\section{EBCT Study}

Postoperative EBCT scanning was performed in all patients on the following schedule: before discharge, 3 or 6 months after the operation, and once each year to evaluate the postoperative time course of the residual false lumen.

The first postoperative EBCT study was made before discharge of each patient (2 to 3 weeks after the operation). In contrast with computed tomographic scans preoperatively (Figure 2, $A$ and $C$, and Figure 3, $A$ and $C$ ), the false lumen in the descending aorta covered by the stented graft closed in all (Figure 2, $B$ and $D$, and Figure 3, $B$ and $D$ ) but 2 chronic cases. In one of these cases the diameter of the false lumen surpassed $65 \mathrm{~mm}$ as a result of a long history, and the 32-mm-diameter stent graft was too small to compact the false lumen. At the diaphragmatic level, the false lumen of the descending aorta distal to the stent graft closed with thrombus in 27 of 29 acute cases and 18 of 21 chronic cases, as shown in Figure 2, D. EBCT follow-up study at 3 

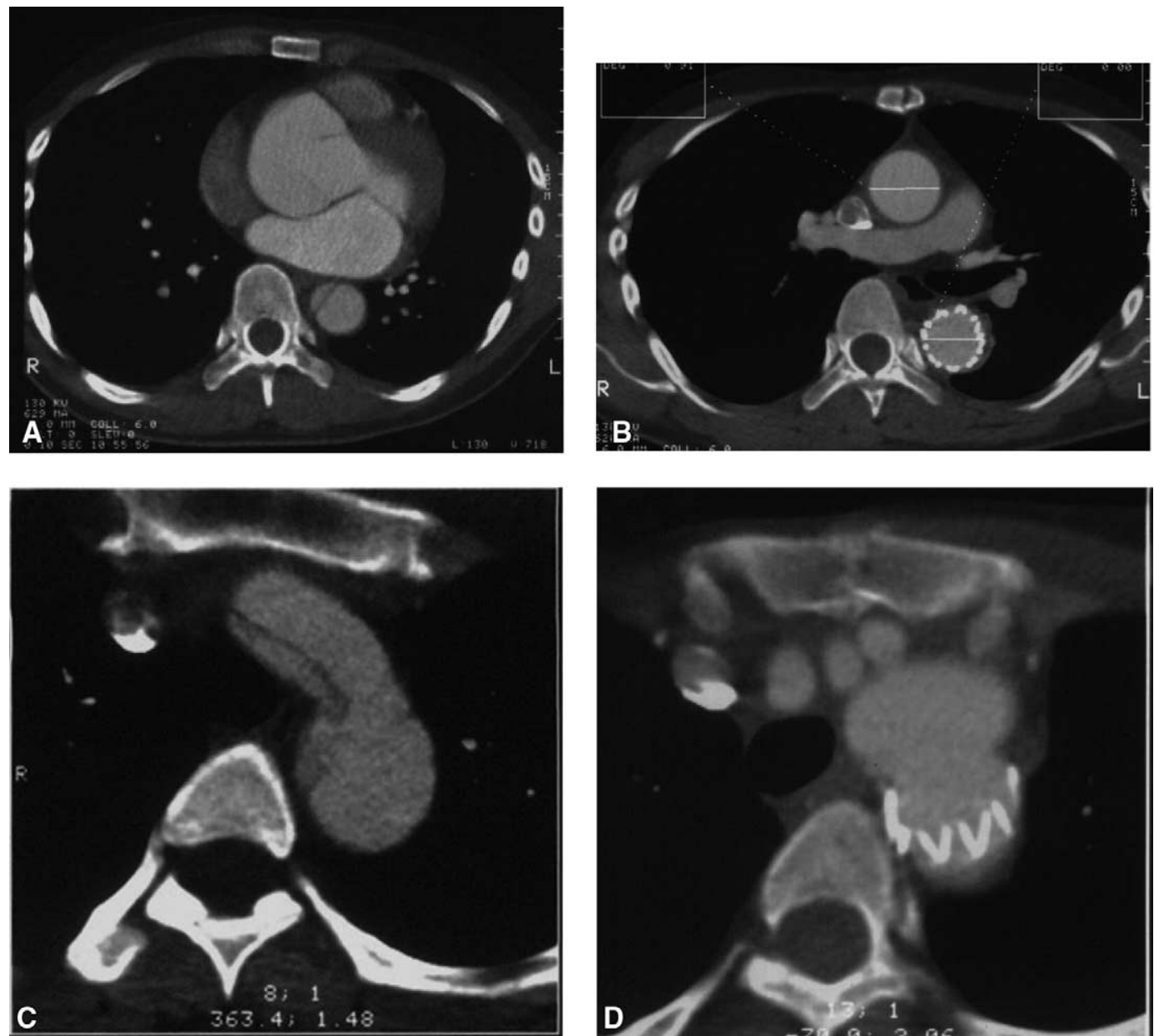

Figure 3. A, Electric beam computed tomography of a patient with Marfan syndrome and acute type A dissection before the operation. The intimal tear is clearly seen in the ascending aorta, and the true lumen in the descending aorta is narrowed severely. B, Electric beam computed tomography of the same patient 6 months after the operation shows the true lumen in the thoracic aorta is restored completely by means of stent graft implantation. C, Electric beam computed tomography shows an acute case in which the primary intimal tear is located in the arch, and the true lumen is severely compressed. D, Electric beam computed tomographic scan 6 months after the operation shows that the arch is restored, and the elastic metal stent is exhibited.

to 6 months postoperatively showed that the residual false lumen in the distal descending aorta remained patent in $8 \%$ of acute and $15 \%$ of chronic cases, respectively. The false lumen of the descending aorta disappeared, and the entire aorta returned to normal in 3 cases. Compared with the data preoperatively, the diameter of the proximal descending aorta was decreased significantly, especially in chronic cases: $28.9 \pm 3.1 \mathrm{~mm}$ preoperatively versus $26.8 \pm 1.3 \mathrm{~mm}(\mathrm{n}=$ 29) 3 months postoperatively in acute cases and $37.6 \pm 7.7$ $\mathrm{mm}$ preoperatively versus $28.8 \pm 5.4 \mathrm{~mm}(\mathrm{n}=21, P<$ .001) 3 months postoperatively in chronic cases. Although a persistent false lumen at the level of the superior mesenteric artery was observed in most patients, no enlargement was noted compared with those before the operation.

\section{Follow-up}

Fifty-eight patients were discharged from the hospital and were followed up in our outpatient clinic or had telephone interviews. Fifty-six patients have resumed normal life with only antihypertensive therapy. The patient who had postoperative paraplegia was able to walk on crutches 4 months after the operation. An EBCT scan is performed at least once each year. There were no late deaths or need for reoperation.

\section{Discussion}

The conventional elephant trunk technique has been widely used for staged aortic replacement. ${ }^{2-4}$ Crawford and associates $^{5}$ reported the complications of this method: kinking and 
occlusion of the graft, paraplegia as a result of clotting around the graft, and peripheral thromboembolism caused by flapping action. They stated that these complications were more likely when the trunk was longer. To avoid these potential complications of a long elephant trunk graft, Kato and coworkers ${ }^{1}$ inserted a graft, the distal part of which was sustained by an intraluminal stent into the descending aorta. Thus any kinking and flapping action of the graft could be prevented. In addition, the dead space around the graft could be completely obliterated.

Ascending aorta and total arch replacement combined with transaortic stent graft implantation has been successfully used in the treatment of acute type A aortic dissection. ${ }^{6,7} \mathrm{We}$ used this method and made some modifications: the whole length of the elephant trunk is sustained by a self-expandable stent, and we call this the skeletonized elephant trunk technique. The role of the stent is to fix the graft to the native aortic wall and make the dissected layers of the wall conglutinate. If the primary intimal tear is located in the descending aorta, where it cannot be reached by means of median sternotomy, the stented graft could seal off the tear and make thrombus obliteration of the downstream false channel possible. In addition, even if the dissection entry point has been eliminated by synthetic graft replacement, anastomotic leakage or a small tear in the suture line of the proximal descending thoracic aorta might exist and cause the false lumen to remain patent and dilated. The stented elephant trunk could prevent this. In most of our cases, including both acute and chronic dissection, the skeletonized elephant trunk implanted into the descending aorta plays a pivotal role in closing the false lumen of the downstream descending thoracic aorta. In our series postoperative thrombus obliteration of the false channel in the descending thoracic aorta sustained by the skeletonized elephant trunk was observed in 33 of 36 acute cases and 20 of 24 chronic cases, suggesting that repair of the total thoracic aorta can be accomplished by using the skeletonized elephant trunk technique. We encountered one chronic dissection in which the diameter of the dissected descending aorta reached 65 $\mathrm{mm}$ and the false channel was extremely expanded. Even though we inserted a large stented graft ( $32 \mathrm{~mm}$ in diameter) into the true lumen of the descending aorta, the false channel was still patent. In this case, in which the false lumen is greater than $50 \mathrm{~mm}$ in diameter, the stent graft could hardly work.

The indications of total arch replacement combined with the skeletonized elephant trunk procedure are Stanford type A aortic dissection with (1) the intimal tear located in the aortic arch or proximal descending aorta (retrograde dissection), (2) serious involvement of the arch vessels, and (3) Marfan disease. Patients with Marfan disease are likely to experience redissection, which will be a disastrous event if the native aortic arch remained and was redissected because there is nowhere for the blood stream in the false lumen to go but to break open the aortic wall. Therefore we insist that total arch replacement is a necessary procedure for the skeletonized elephant trunk technique. It is not a good indication to perform the skeletonized elephant trunk procedure for those chronic cases with aneurysms surpassing $50 \mathrm{~mm}$ in diameter. In these cases 1- or 2-stage total aortic replacement should be recommended.

We have routinely used right axillary artery cannulation for both extracorporeal circulation and antegrade SCP in type A dissection cases since $1996^{8}$ because the femoral artery is frequently involved by dissection and femoral artery cannulation and might cause malperfusion of some vital organs. We found that the axillary artery is rarely involved in dissection, through which the arterial perfusion is antegrade and physiologic. Our experiences in more than 300 cases have proved that CPB by way of right axillary artery cannulation is feasible and convenient.

Neurologic injury is one of the major complications in the repair of the transverse aortic arch, irrespective of whatever protective measures have been taken. ${ }^{9}$ We have routinely used hypothermic antegrade SCP for brain protection since 1996. The morbidity of cerebral disorders in our patient population is low compared with that in the literature. Three reasons might contribute to this consequence. First, our patients are relatively young, and the atherosclerotic changes in the aortic wall are not severe. Second, the antegrade SCP technique we used is a more physiologic and effective means of cerebral protection. Finally, the average SCP and lower body arrest time in our series are short. Making sure that the Willis circle is intact is crucial when using the SCP technique. We monitored the blood pressure of the left common carotid artery during the operation. If the pressure is lower than $20 \mathrm{~mm} \mathrm{Hg}$ during SCP at a perfusion rate of $10 \mathrm{~mL} \cdot \mathrm{kg}^{-1} \cdot \mathrm{min}^{-1}$, we control the SCP time within 30 minutes under deep hypothermia. We encountered one case in which the blood pressure of the left common carotid artery was undetectable, which suggested the Willis circles were hypoplastic in this patient. We spent 45 minutes on the arch replacement and stented graft implantation during SCP, which proved too long to be tolerated. This patient exhibited right-side hemiplegia, and postoperative computed tomographic scanning showed ischemic change of the left-side cerebral hemisphere. Transcranial color Doppler flow imaging is also a useful measure in detection of cerebral blood flow during SCP and should be recommended.

Ischemic spinal cord damage is the most serious complication associated with the skeletonized elephant trunk procedure. ${ }^{6}$ To prevent the vital intercostal arteries below the Th8 level being occluded by the stented graft, the distal ends of the implanted stent grafts were located between the Th7 and Th8 levels, as evaluated by means of postoperative 
chest radiography. In our series postoperative paraplegia was observed only in one case, in which the lower body arrest time was 72 minutes at a temperature of $18^{\circ} \mathrm{C}$ to $20^{\circ} \mathrm{C}$ (bladder temperature), which was much longer than the mean of $24 \pm 13$ minutes. The prolonged ischemic time could be the main reason for spinal cord injury in this patient.

There were 2 in-hospital deaths in our group. The causes of death were extensive cerebral infarction in one case and multiorgan and system failure in another. We noticed that all the patients who exhibited cerebral infarction postoperatively had a history of preoperative cerebral infarction, and those who had acute renal failure postoperatively had a history of acute renal dysfunction preoperatively. Acute renal dysfunction and a history of cerebral infarction might be risk factors of this aggressive procedure.

The EBCT follow-up study 3 months after the operation in the present series demonstrated that thrombus obliteration of the false lumen of the descending aorta distal to the skeletonized elephant trunk was observed in 27 of 29 acute cases and 18 of 21 chronic cases at the diaphragmatic level. In most cases of acute and chronic type A aortic dissection, the goal of complete thoracic aortic repair has been achieved with the skeletonized elephant trunk technique.

\section{Conclusion}

The skeletonized elephant trunk procedure is a feasible treatment for both acute and chronic type A aortic dissection. By using this method, the goal of total thoracic aortic repair could be achieved, and a second operation in the distal descending aorta might become less necessary.

\section{References}

1. Kato M, Ohnishi K, Kaneko M, Ueda T, Kishi D, Mizushima T, et al. New graft-implanting method for thoracic aortic aneurysm or dissection with a stented graft. Circulation. 1996;94(suppl II):II188-93.

2. Borst HG, Walterbusch G, Schaps D. Extensive aortic replacement using the "elephant trunk" prosthesis. Thorac Cardiovasc Surg. 1983; 31:37-40.

3. Heinemann MK, Buehner B, Jurmann MJ, Borst HG. Use of the "elephant trunk technique" in aortic surgery. Ann Thorac Surg. 1995; 60:2-7.

4. Svensson LG. Rationale and technique for replacement of the ascending aorta, arch, and distal aorta using a modified elephant trunk procedure. J Card Surg. 1992;7:301-21.

5. Crawford ES, Coselli JS, Svensson LG, Safi HJ, Hess KR. Diffuse aneurysmal disease (chronic aortic dissection, Marfan, and mega aorta syndromes) and multiple aneurysm: treatment by subtotal and total aortic replacement emphasizing the elephant trunk operation. Ann Surg. 1990;211:521-37.

6. Mizunoa T, Toyamab M, Tabuchi N, Wu H, Sunanori M. Stented elephant trunk procedure combined with ascending aorta and arch replacement for acute type A aortic dissection. Eur J Cardiothorac Surg. 2002;22:504-9.

7. Ishihara H, Uchida N, Yamasaki N, Sakashita M, Kanou M. Extensive primary repair of the thoracic aorta in Stanford type A acute aortic dissection by means of a synthetic vascular graft with self-expandable stent. J Thorac Cardiovasc Surg. 2002;123:1035-40.
8. Sun L, Ren J, Yang T, Chang Q, Zhu J. Surgical treatment of DeBakey-I aortic dissection using the "elephant trunk technique." Chin Med J. 1999;112:713-6.

9. Bavaria J, Brinster D, Gorman R, Woo Y, Gleason T, Pochettino A. Advances in the treatment of acute type A dissection: an integrated approach. Ann Thorac Surg. 2002;74(suppl):S1848-52.

\section{Discussion}

Dr R. Scott Mitchell (Stanford, Calif). Dr Liu, congratulations on a very nice presentation and really an excellent series: 60 dissections in 15 months is more than one could hope for. These results are really quite excellent; a 3\% total mortality and, according to your definitions, $92 \%$ of your acute patients had complete obliteration of the false lumen and $85 \%$ in the chronic patients. Therefore it is indeed perhaps attractive as a single-stage approach to the entire thoracic aorta. An important detail is that your elephant trunk is only $10 \mathrm{~cm}$ long, and that is important because other investigators have noted that if you extend that graft too far distally, you will increase the rate of paraplegia.

One of the difficulties with new technology is this: Just because you can do something, should you do something? Therefore it is not entirely clear what your indications were, especially in chronic dissections. Certainly for acute dissections, if you can reverse malperfusion, that would be very good, but in chronic dissections it concerns me that you might create malperfusion. Therefore that really leads me to my 2 questions.

For acute type A dissections, did you have malperfusion that you successfully reversed with the elephant trunk?

Dr Liu. Thank you, Dr Mitchell. That is a very good question. Fortunately, we did not encounter this kind of situation. We did not encounter malperfusion of the lower body. The indications for those chronic aortic dissections we have discussed in the slides. Not all kinds of chronic dissections were appropriate for this procedure. The diameter of the descending aorta surpassing $50 \mathrm{~cm}$ is not a good indication; a stent graft cannot work well in this situation.

Dr Mitchell. I noticed in one of your slides that you had true lumen collapse, and in the follow-up computed tomogram, there was very nice approximation, so that it appeared in the acute situation that indeed you could significantly improve the morphology. I guess my major concern is for the chronic dissections. If you redirect all the flow into the true lumen, you might not perfuse vessels from the false lumen. Therefore my question then for the chronic cases is this: Did you see paraplegia, perhaps that was related to cord flow from the false lumen, or did you see abdominal visceral ischemia from false lumen vessels that were not perfused after your elephant trunk?

Dr Liu. Thank you, Dr Mitchell. In chronic cases we did not have any complications associated with the skeletonized elephant trunk procedure. In fact, we had one paraplegic patient who was in the acute aortic dissection group. The cause might be clot formation in the false lumen in which the intercostal artery arises, and that is the reason, we suppose.

Dr Ludwig K. von Segesser (Lausanne, Switzerland). Can you tell us how you size your covered stent graft?

Dr Liu. We measured the diameter before the operation in a computed tomographic photo and decided what we would use and which size was best, and also we examined this during the operation. 